\title{
Selección de un material alternativo para el diseño y manufactura de cuchillas del motocultor YTO DF151, para trabajos en suelos de la región centro del Ecuador.

Selection of an alternative material for the design and manufacture of knives on the motocultor YTO DF151, for work on soils of the central region of Ecuador.

Patricio Abarca Pérez. ${ }^{1}$, Marcelo Castillo Cárdenas. ${ }^{2}$, David Bravo Morocho. ${ }^{3}$, Fabián Sánchez Carrión. ${ }^{4} \&$ Paúl Motúfar Paz. ${ }^{5}$

Abstract.

DOI: https://doi.org/10.33262/cienciadigital.v2i3.170

The objective of this study is to select a material that replaces and improves the performance shown by the steel AISI 1030, used for the manufacture of the blades of tillage of the YTO DF 15I tractors of Chinese origin, intended for work in the soils of region's center of Ecuador. AISI 1030 steel presents a low reliability for this type of applications. The material selection procedure is based on the method proposed by ASHBY. The design requirements of the blades, consider restrictions for the material, such as: minimum specified yield strength, specified hardness, feasibility of being forged and feasibility of being thermally welded. In addition, the objective is to maximize the fracture tenacity and, of course, minimize the price of the material; to achieve that, a material index is used to identify the best performing materials, which optimizes the selection. With the help of a material selection software, it was possible to debug all available information, and it turned out that the low alloy steel AISI 4135, hardened and tempered, could be a strong candidate to replace the AISI 1030 steel, since, apart from to comply with all the restrictions required for the design, it

\footnotetext{
${ }^{1}$ Escuela Superior Politécnica de Chimborazo, Chimborazo, Ecuador, edison.abarca@espoch.edu.ec

${ }^{2}$ Escuela Superior Politécnica de Chimborazo, Chimborazo, Ecuador, edison.castillo@espoch.edu.ec

${ }^{3}$ Escuela Superior Politécnica de Chimborazo, Chimborazo, Ecuador, victor.bravo@espoch.edu.ec

${ }^{4}$ Escuela Superior Politécnica de Chimborazo, Chimborazo, Ecuador, esanchez_c@espoch.edu.ec

${ }^{5}$ Escuela Superior Politécnica de Chimborazo, Chimborazo, Ecuador, paul.montufar@espoch.edu.ec
} 
also has the highest value of fracture/price tenacity ratio $\left(K_{I c} / P=200\right)$.

Keywords: Material Selection, Tillage Blades, Performance, Reliability, Material Index.

\section{Resumen.}

El objetivo de este estudio es seleccionar un material que reemplace y mejore el rendimiento mostrado por el acero AISI 1030, utilizado para la manufactura de las cuchillas de labranza o aspas del motocultor YTO DF 15I de origen chino, destinado para trabajos en suelos de la región centro del Ecuador. El acero AISI 1030 presenta una baja fiabilidad para este tipo de aplicaciones. El procedimiento de selección del material se fundamenta en el método propuesto por ASHBY. Los requerimientos de diseño de las cuchillas empleadas en el motocultor, consideran restricciones para el material, tales como: límite de fluencia mínimo especificado, dureza especificada, factibilidad de ser forjado y factibilidad de ser soldado térmicamente. Además, el objetivo es maximizar la tenacidad a la fractura y por supuesto, minimizar el precio del material; para lograr aquello, se utiliza un índice de material que permite identificar los materiales de mejor desempeño, con lo cual se optimiza la selección. Con la ayuda de un software de selección de materiales, se logró depurar toda la información disponible, dando como resultado que el acero de baja aleación AISI 4135 templado y revenido, podría ser un firme candidato para reemplazar al acero AISI 1030, puesto que, aparte de cumplir con todas las restricciones requeridas para el diseño, también cuenta con el valor más alto de relación tenacidad a la fractura/precio $\left(K_{I c} / P=200\right)$.

Palabras Claves: Selección de Materiales, Cuchillas de Labranza, Rendimiento, Fiabilidad, Índice de Material.

\section{Introducción .}

El motocultor es considerado como un tractor de un solo eje, el cual es conducido por medio de unos brazos o manceras, mientras el operador camina sobre el terreno. Su funcionamiento se basa en el arado rotativo (rotavator o rotavación), en donde cuchillas o aspas de labranza dispuestas simétricamente alrededor de un eje (el cual es accionado por la toma de potencia del motocultor), giran con movimiento circular, de tal manera que arranca, corta y mezcla el perfil del terreno sobre el cual trabajan (Gonzales et al., 2007). Uno de los problemas más significativos en la utilización de arados rotativos, es la fractura de las cuchillas cuando las mismas impactan con rocas y/o suelos duros, lo que obliga generalmente a emplear sistemas de embrague que mitigan el impacto, de esta manera se disminuye el riesgo de fractura de las cuchillas debido a las sobrecargas (Wilkinson \& 
Braunbeck, 1977).

Las cuchillas, a parte de los elevados niveles de esfuerzos que le ocasionan deformación plástica, también se exponen al contacto permanente con partículas duras y/o abrasivas, que le causan desprendimiento de material, estos dos parámetros son una de las principales causas de falla y pérdida de desempeño de las cuchillas (Hutchings, 1992). En general, los aceros son el material básico de las cuchillas de labranza, los cuales deben poseer una adecuada combinación de tenacidad a la fractura y resistencia al desgaste (Gonzales et al., 2007), dichas propiedades mecánicas variarán según su microestructura y composición química (ASTM, 1987).

\section{Importancia del problema.}

Actualmente en el Ecuador no existe un documento específico, que regule las exigencias mínimas para la adquisición de herramientas de labranza agrícola, de tal manera que sea un apoyo técnico para prevenir la falla prematura de estos elementos. Las cuchillas o aspas son un caso particular de esta problemática, lo que ocasiona paros perjudiciales en las tareas agrícolas, y consecuentemente pérdidas económicas y de producción (Castillo \& Bravo, 2017).

Las cuchillas de labranza o aspas utilizadas en el motocultor YTO DF 15I de origen chino, son fabricadas de acero AISI 1030, y presentan un serio inconveniente, su índice de fiabilidad es bajo, esto se justifica puesto que un 30 a $40 \%$ de estas, sufren fracturas en su base, lo que desencadena en una serie de problemas en el desempeño del motocultor y por ende malestar en el operador, puesto que las áreas de terreno por donde pasan las cuchillas rotas, quedan enteras o sin labrar (Castillo \& Bravo, 2017).

Uno de los parámetros más importantes para atenuar la falla de las cuchillas y extender su vida útil, tiene que ver con el diseño de las mismas, y dentro de este, la selección del material con el cual serán manufacturadas, juega un papel determinante. Por lo referido, en la presente investigación se expone un estudio acerca de la selección de los posibles materiales o metales que podrían sustituir al ya aplicado (acero AISI 1030), y que permitan superar la fiabilidad de las cuchillas actualmente utilizadas.

\section{Metodología.}

Para seleccionar los materiales o metales más idóneos que superen en rendimiento al metal vigente (acero AISI 1030) en las aspas, se implementará el método de Ashby que a continuación se especifica.

Método de selección de materiales propuesto por Ashby. 
El procedimiento básico para la selección de un material, es establecer el vínculo entre el material y la función del elemento. Un material tiene atributos (propiedades), tales como: su densidad, resistencia mecánica, costo, resistencia a la corrosión, entre otros. Un diseño exige cierto requerimiento de estos: una baja densidad, una alta resistencia, un bajo costo y resistencia a algún ambiente en especial. La selección de materiales implica buscar la mejor combinación estos requisitos de diseño, de tal forma que satisfagan adecuadamente la aplicación para la cual fueron diseñados (Ashby, 2011). Las etapas para seleccionar un material son los siguientes:

\section{Interpretación.}

Es el primer paso para abordar la selección de un material, aquí se traducen los requisitos de diseño del componente (se define lo que debe hacer) en una descripción para el material, se definen las condiciones de contorno para poder seleccionarlo. La etapa de interpretación define los siguientes ítems (Ashby, 2011):

La función: Cualquier componente de ingeniería tiene una o más funciones: soportar una carga, contener una presión, transmitir calor, entre otros.

Restricciones: Un elemento tiene restricciones, tales como: que la geometría sea fija, que el componente resista cargas o presiones sin falla, que sea aislante o conductor, que trabaje en un cierto rango de temperatura y en un ambiente dado, entre otros.

Objetivo: El trabajo de diseño involucra un objetivo, por ejemplo, hacer que el elemento sea tan ligero como sea posible, o que sea muy barato, o una combinación de estos, entre otros.

Variables libres: El diseñador puede ajustar ciertos parámetros, por ejemplo, puede variar las dimensiones que no interfieran con los requisitos de diseño, y lo que es más importante, puede elegir libremente el material para el componente.

\section{Filtración: límites de atributos.}

La selección imparcial requiere que todos los materiales se consideren candidatos hasta que se demuestre lo contrario. Por tanto, se debe realizar una filtración, es decir, se elimina a los candidatos en los cuales uno o más de sus atributos se encuentra fuera de los límites establecidos por las restricciones (Ashby, 2011).

\section{Clasificación: índices de materiales.}

Los materiales que han pasado la etapa de filtración, se los clasifica para determinar cuáles de ellos pueden tener el mejor desempeño, para lo cual es necesario aplicar criterios de optimización más conocidos como índices de materiales. La propiedad o el grupo de 
propiedades que maximiza el rendimiento para un diseño se denomina índice de material. Los índices brindan criterios de excelencia que permiten clasificar los materiales, según su capacidad para un buen desempeño en la aplicación determinada. El maximizar o minimizar una sola propiedad puede maximizar el rendimiento, pero generalmente el rendimiento está limitado no por una sola propiedad, sino por una combinación de ellas, por ejemplo, los mejores materiales para un elemento fuerte y ligero, son aquellos con el mayor valor de resistencia específica, es decir, la mayor relación $\sigma \mathrm{f} / \rho$, donde $\sigma \mathrm{f}$ es la resistencia a la falla y $\rho$ es la densidad. Hay muchos índices de este tipo, cada uno asociado con la maximización de algún aspecto del rendimiento (Ashby, 2011).

\section{Equipos y materiales utilizados.}

Para facilitar o simplificar el estudio, se llevará a cabo una selección asistida por computadora, en donde el software de selección y procesos contiene una base de datos actualizada, es decir un registro completo de materiales aplicados en ingeniería. Cada registro contiene datos de atributos (propiedades) estructurados para un material en particular, y cada atributo (propiedad) contempla un rango típico (o a menudo su rango permitido).

\section{Selección del material para las aspas.}

Antes de proceder a la selección de un material alternativo que incremente el índice de fiabilidad de las aspas, es necesario presentar los datos técnicos del acero AISI 1030 al cual se busca reemplazar y superar en rendimiento, tales datos se presentan a continuación en la Tabla 1.

Tabla 1 Propiedades del acero AISI 1030, material actual de las cuchillas del motocultor YTO DF151.

\begin{tabular}{ll}
\hline Propiedades físicas & Valor \\
\hline $\begin{array}{l}\text { Densidad } \\
\text { Propiedades mecánicas }\end{array}$ & $7.85 \mathrm{~g} / \mathrm{cm}^{3}$ \\
Dureza & $47.5 \mathrm{HRC}$ \\
Resistencia última a la tracción & $525 \mathrm{MPa}$ \\
Resistencia a la fluencia & $345 \mathrm{MPa}$ \\
Porcentaje de elongación & $32 \%$ \\
Módulo de elasticidad & $206 \mathrm{GPa}$ \\
Razón de poisson & 0.29 \\
\hline
\end{tabular}

Fuente: (Castillo \& Bravo, 2017).

Seguidamente, de acuerdo a la metodología planteada, se inicia el proceso de selección.

\section{La Interpretación.}

Mecánicamente hablando, el aspa se comporta como una viga en voladizo expuesta a una carga distribuida de 3,6 N/mm que le produce flexión (Castillo \& Bravo, 2017) (Figura 1). Esta carga es simulada en un software de elemento finito, de donde se obtiene que el 
máximo esfuerzo equivalente de Von - Misses es de $\sigma^{\prime}=156,46 \mathrm{MPa}$ (Figura 1).

Figura 1. Simulación de carga distribuida aplicada a las aspas del motocultor YTO DF151.

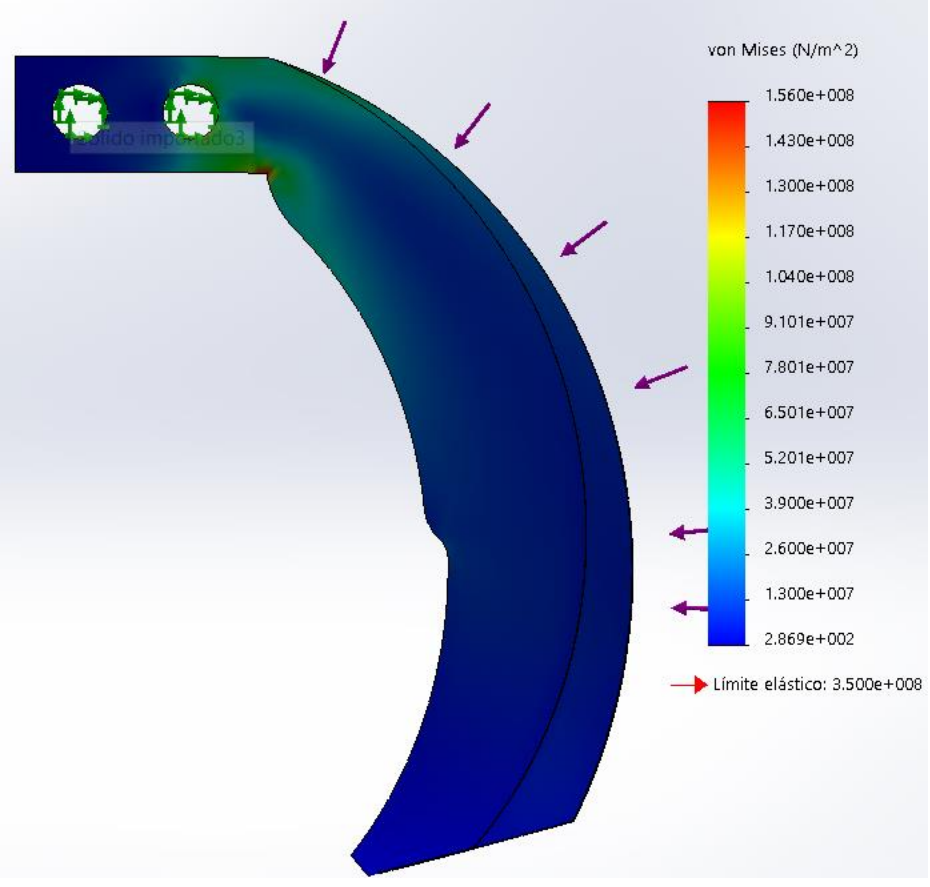

Fuente: (Autores).

El criterio del esfuerzo equivalente de Von-Misses (Budynas \& Nisbett, 2012) se define como:

$$
\sigma^{\prime} \leq \frac{S_{y}}{n}
$$

Donde:

$$
\begin{aligned}
& \sigma^{\prime}=\text { Esfuerzo equivalente de Von }- \text { Misses } \\
& S_{y}=\text { Límite de fluencia del material }
\end{aligned}
$$

Puesto que la geometría de la cuchilla tiene que ser fija, y para un factor de seguridad de 2 (Budynas \& Nisbett, 2012), el límite de fluencia mínimo que se requiere para el material sería:

$$
\sigma^{\prime} * n \leq S_{y}
$$

$$
156,46 * 2 \leq S_{y}
$$




$$
S_{y} \geq 312,96 \times 10^{6} \mathrm{~Pa}
$$

El material de las aspas debe también poseer una adecuada combinación de tenacidad a la fractura y resistencia al desgaste (Muñoz, 2000).

Los requerimientos básicos para un implemento agrícola, en este caso las aspas que se desenvuelven en suelo normal, indican que la dureza del material debe estar en el rango de 38 - 45 HRC (363 - 454 HV) (Cañavate, 2014).

Puesto que los motocultores se desenvuelven en comunidades indígenas, el precio del material con el cual serán manufacturadas las cuchillas es muy importante, debido a que son comunidades de escasos recursos económicos.

Otro aspecto muy importante es el proceso de manufactura de las cuchillas, y para ello es condicionante que el material tenga la factibilidad de ser forjado y, de darse el caso, sea idóneo para soldadura mediante algún procedimiento térmico.

Los requerimientos de diseño para una cuchilla se resumen en la Tabla 2.

Tabla 2 Requerimientos de diseño para las cuchillas utilizadas en el motocultor YTO DF151.

\begin{tabular}{ll}
\hline Función & Cuchilla o aspa - viga rígida \\
\hline Restricciones & Geometría especificada \\
& Límite de fluencia mínimo especificado \\
& Dureza especificada \\
& Factible de forja \\
& Factible de soldadura térmica \\
\hline Objetivo & Maximizar la tenacidad a la fractura y \\
& minimizar el precio \\
\hline Variables libres & Elección del material \\
\hline
\end{tabular}

\section{Filtración: límites de atributos.}

En el software de selección se ingresa las restricciones del material ya definidas, dando como resultado que de todo el universo de materiales (3905 materiales de ingeniería posibles), solo 267 pasaron el filtro, y estos pertenecen a la familia de metales y aleaciones metálicas, tal resultado se expone en la Figura 2. Aunque sólo se muestra los resultados para las condiciones acerca del límite de fluencia mínimo y rango de dureza, los requisitos de que el material también pueda ser forjado y soldado ya están involucrados en dicho filtro.

Figura 2. Etapa de filtración, en dónde solo 267 materiales (que resultan ser sólo metales y aleaciones metálicas) de 3905 pasaron las restricciones de diseño: a) Gráfico de barras en donde se muestra la filtración en función del rango de dureza Vickers, b) Gráfico de barras en donde se muestra la filtración en función del mínimo límite de fluencia permitido. Nota: 
Los requerimientos acerca de que el material pueda exponerse a la forja y a la soldadura ya están involucrados en dicho filtro.

a)

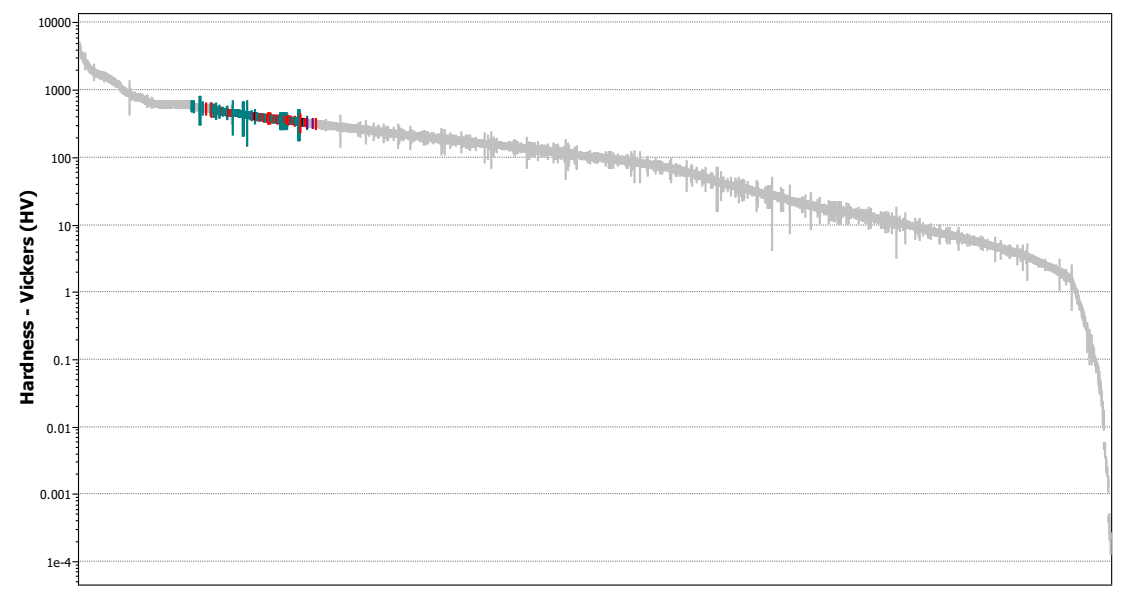

b)

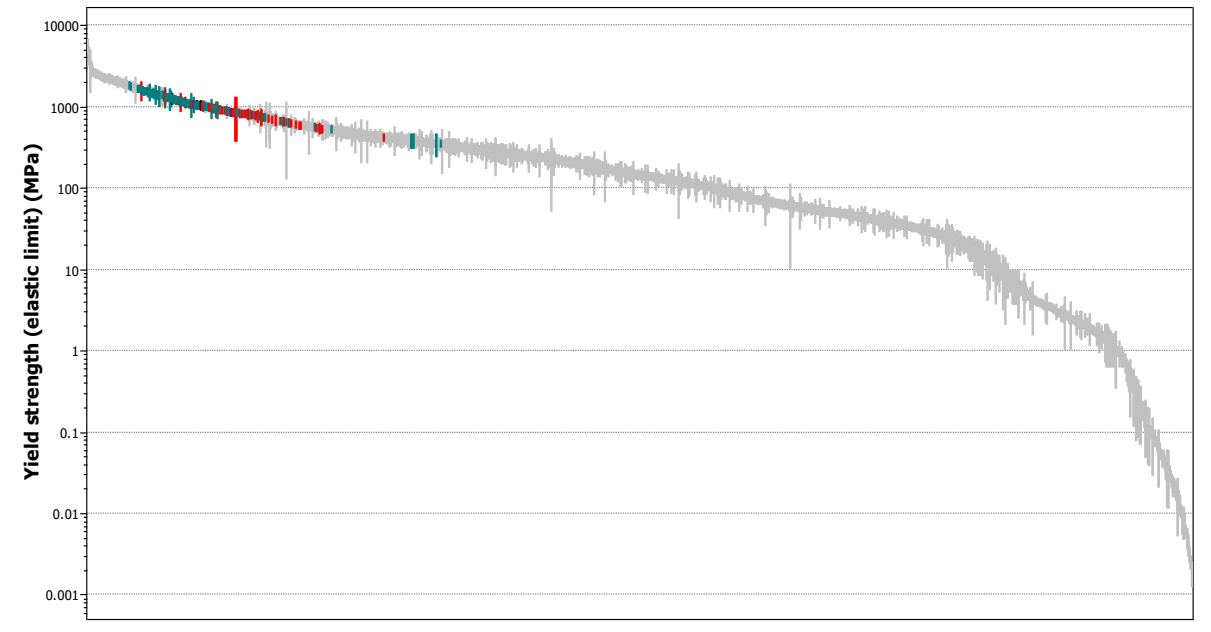

Fuente: (Autores).

Como se había mencionado, el precio y la tenacidad a la fractura tienen mucha influencia para la selección del material, es por eso que evaluando el Gráfico 3, se puede ejecutar un nuevo filtro.

Se puede evidenciar que las aleaciones especiales (burbujas de color rojo), además de cumplir con las restricciones, también poseen los más altos niveles de tenacidad a la fractura, pero su precio sale del alcance económico para poder adquirir el material.

En cambio, los metales ferrosos (burbujas de color celeste) ofrecen una mejor condición de ahorro y sin descuidar la tenacidad a la fractura que sigue siendo relativamente buena. 
Es por eso que el siguiente filtro (Figura 4) se basa en seleccionar únicamente a los metales ferrosos, dando como resultado que, de 267 materiales, ahora quedan 178 candidatos posibles.

Se nota también que en este grupo ya aparece el acero AISI 1030 (burbuja de color amarillo) que es el material actual de las cuchillas y a quien se desea reemplazar y superar en rendimiento.

Figura 3. Gráfico de burbujas Precio vs Tenacidad a la fractura de aquellos materiales que pasaron las restricciones.

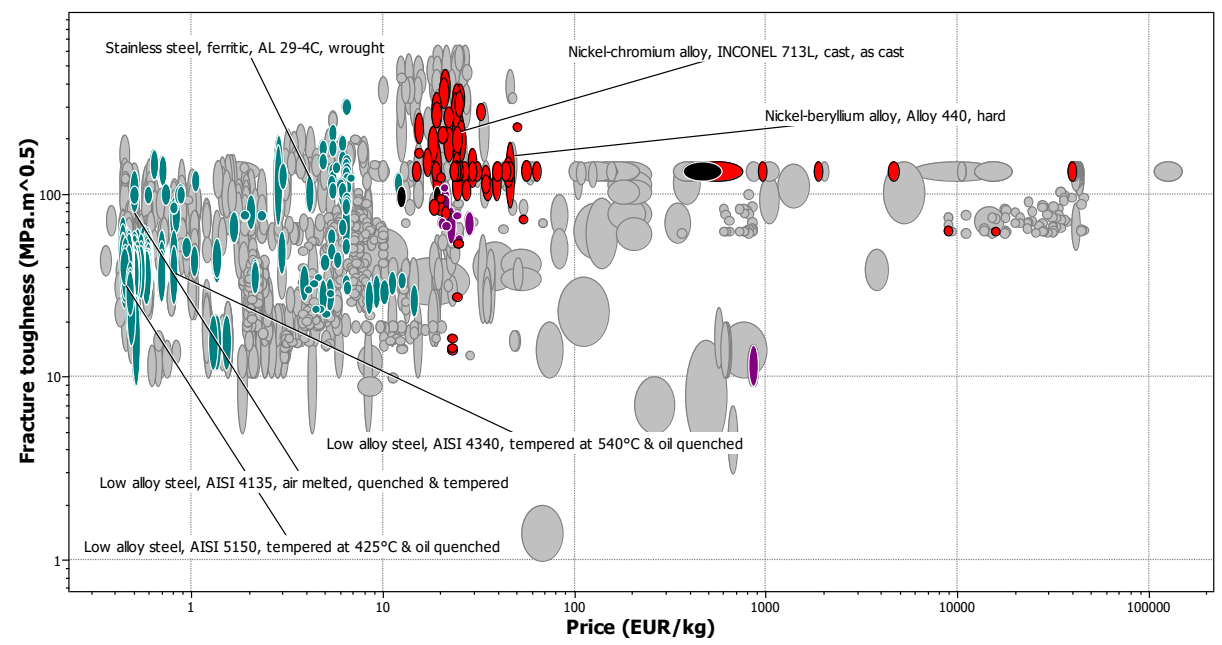

Figura 4. Gráfico de burbujas Precio vs Tenacidad a la fractura, sólo de metales ferrosos, puesto que presentan una mayor factibilidad económica, en donde se ha reducido de 267 a 178 candidatos posibles. Aquí ya aparece el acero AISI 1030 (burbuja amarilla), material actual de las cuchillas

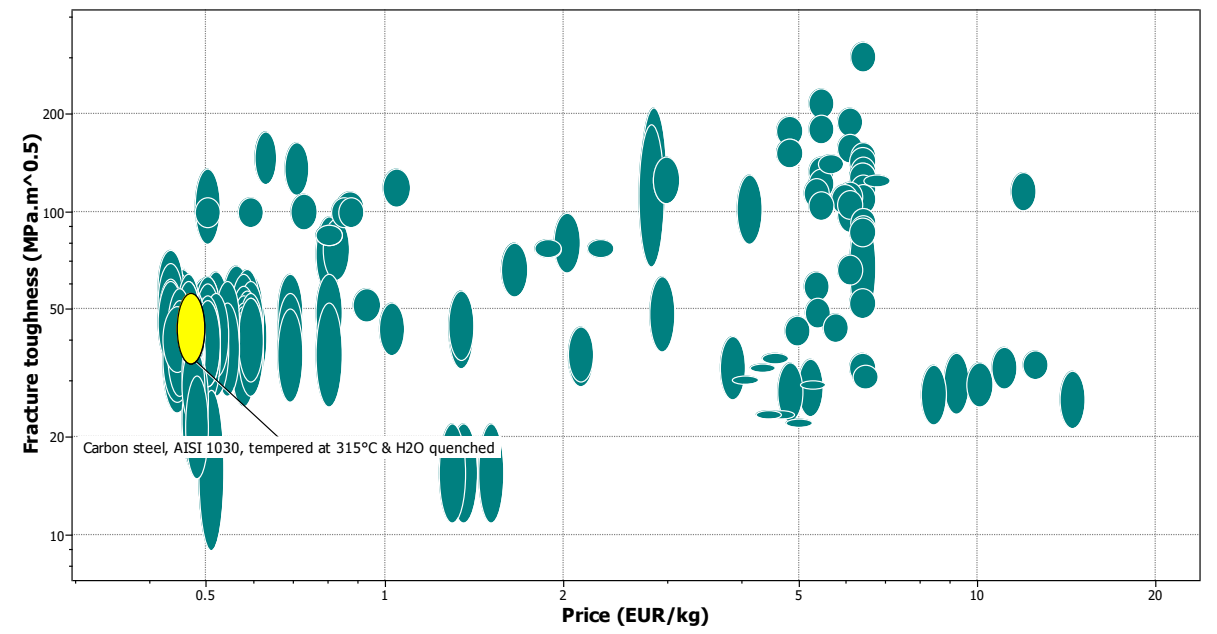




\section{Clasificación: índice del material .}

Una vez que los metales ferrosos han sido determinados como el grupo de materiales que han pasado las restricciones, y que paralelamente también se podrían ajustar a la viabilidad económica disponible, ahora se buscará identificar cuáles de ellos tendrán el mejor desempeño. El objetivo del diseño es ahora, maximizar la tenacidad a la fractura y, por supuesto, minimizar el precio. Por lo tanto, el índice de material que permitirá evaluar este requerimiento es el siguiente:

$$
M=\frac{K_{I c}^{0.5}}{P}
$$

Donde: $\quad M=$ Índice del material

$$
K_{I c}=\text { Tenacidad a la fractura }
$$

Si se despeja la tenacidad a la fractura de la ecuación 2, y se aplica logaritmos a ambos miembros, se tiene:

$$
\begin{aligned}
\frac{1}{2} K_{I c} & =\log P+\log M \\
K_{I c} & =2 \log P+2 \log M
\end{aligned}
$$

La ecuación 3 es análoga a la recta $y=m x+b$, y representará una línea guía de selección de pendiente 2, que ubicada en el gráfico Precio vs Tenacidad a la fractura, buscará minimizar el precio del material, y de esta manera optimizar la selección final. Puesto que el objetivo es superar en rendimiento al acero AISI 1030, la línea de selección guía de pendiente 2, se ubica por encima de dicho acero, de tal forma que sobresalgan sólo aquellos aceros con mayor relación $K_{I c} / P$. Con este nuevo criterio, el número de candidatos que hasta ahora era de 178, se logra reducir a 31 (Figura 5).

Figura 5. Gráfico de burbujas Precio vs Tenacidad a la fractura, en donde la línea guía de selección ha logrado depurar sólo aquellos metales ferrosos que superan en rendimiento (relación $K_{I C} / P$ ) al acero AISI 1030 (burbuja amarilla), reduciendo el número de candidatos desde 178 hasta 31.

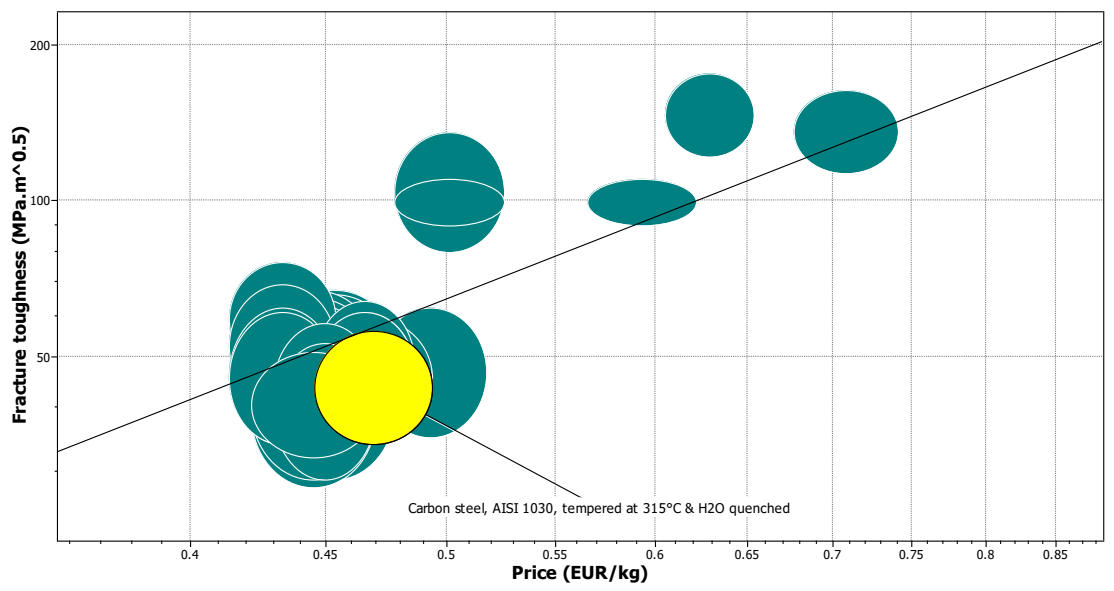




\section{Análisis de Resultados.}

La línea guía de selección elimina aquellos materiales que estén por debajo y que tampoco intersequen a la misma. A pesar de que el número de candidatos se había reducido a 31, únicamente se van a considerar a aquellos que estén total o al menos más del $50 \%$ por encima de la línea guía. Mediante esta consideración ahora la lista final de candidatos se reduce a 11 (Tabla 3 ).

Tabla 3 Lista de materiales ferrosos, potenciales a reemplazar al acero AISI 1030, en donde se expone la relación $K_{I c} / P$ ordenada de menor a mayor para una mejor apreciación.

\begin{tabular}{|c|c|c|c|c|c|c|}
\hline Denominación & Clasificación & Estado & $\begin{array}{l}\text { Límite } \\
\text { elástico } \\
(\text { Mpa })\end{array}$ & $\begin{array}{c}\text { Precio } \\
\text { promedio } \\
(\text { Euro/kg) }\end{array}$ & $\begin{array}{c}\text { Tenacidad a } \\
\text { la fractura } \\
\text { promedio } \\
\left(M p a^{*} m^{\wedge} 0,5\right)\end{array}$ & $\begin{array}{c}\text { Relación } \\
\text { Tenacidad/precio }\end{array}$ \\
\hline AISI 1030 & $\begin{array}{l}\text { Acero al } \\
\text { carbono }\end{array}$ & $\begin{array}{l}\text { Revenido a } 205^{\circ} \mathrm{C} \\
\text { y templado en agua }\end{array}$ & $\begin{array}{c}580- \\
715\end{array}$ & 0,47 & 42 & 89,4 \\
\hline AISI 1030 & $\begin{array}{l}\text { Acero al } \\
\text { carbono }\end{array}$ & $\begin{array}{l}\text { Templado a } 315^{\circ} \mathrm{C} \\
\text { y templado en agua }\end{array}$ & $\begin{array}{c}555- \\
685\end{array}$ & 0,47 & 42 & 89,4 \\
\hline AISI 1095 & $\begin{array}{l}\text { Acero al } \\
\text { carbono }\end{array}$ & $\begin{array}{c}\text { Templado a } 425^{\circ} \mathrm{C} \\
\text { y templado en } \\
\text { aceite }\end{array}$ & $690-855$ & 0,47 & 49 & 104,3 \\
\hline AISI 5150 & $\begin{array}{c}\text { Acero de } \\
\text { baja aleación }\end{array}$ & $\begin{array}{c}\text { Revenido a } 540{ }^{\circ} \mathrm{C} \\
\text { y templado en } \\
\text { aceite }\end{array}$ & $\begin{array}{l}925- \\
1,14 \mathrm{e} 3\end{array}$ & 0,445 & 50 & 112,4 \\
\hline AISI 5160 & $\begin{array}{c}\text { Acero de } \\
\text { baja aleación }\end{array}$ & $\begin{array}{l}\text { revenido a } 540{ }^{\circ} \mathrm{C} \mathrm{y} \\
\text { templado en aceite }\end{array}$ & $\begin{array}{c}935- \\
1,15 \mathrm{e} 3\end{array}$ & 0,445 & 55 & 123,6 \\
\hline AISI 5046 & $\begin{array}{c}\text { Acero de } \\
\text { baja aleación }\end{array}$ & $\begin{array}{c}\text { Revenido a } 315^{\circ} \mathrm{C} \\
\text { y templado en } \\
\text { aceite }\end{array}$ & $\begin{array}{l}1,04 \mathrm{e} 3- \\
1,28 \mathrm{e} 3\end{array}$ & 0,43 & 55 & 127,9 \\
\hline AISI 5046 & $\begin{array}{c}\text { Acero de } \\
\text { baja aleación }\end{array}$ & $\begin{array}{c}\text { Revenido a } 425^{\circ} \mathrm{C} \\
\text { y templado en } \\
\text { aceite }\end{array}$ & $\begin{array}{c}840- \\
1,02 \mathrm{e} 3\end{array}$ & 0,43 & 60 & 139,5 \\
\hline AISI 8735 & $\begin{array}{c}\text { Acero de } \\
\text { baja aleación }\end{array}$ & Normalizado & $\begin{array}{c}483- \\
534\end{array}$ & 0,59 & 100 & 169,5 \\
\hline YS1200 & $\begin{array}{c}\text { Acero } \\
\text { matensítico }\end{array}$ & $\begin{array}{l}\text { Laminado en } \\
\text { caliente }\end{array}$ & $\begin{array}{c}900- \\
1,15 \mathrm{e} 3\end{array}$ & 0,71 & 140 & 197,2 \\
\hline
\end{tabular}




\begin{tabular}{|c|c|c|c|c|c|}
\hline AISI 4135 & $\begin{array}{c}\text { Acero de } \\
\text { baja aleación }\end{array}$ & Normalizado & $\begin{array}{c}483- \\
586\end{array}$ & 0,5 & 100 \\
\hline AISI 4135 & $\begin{array}{c}\text { Acero de } \\
\text { baja aleación }\end{array}$ & $\begin{array}{l}\text { Templado y } \\
\text { revenido }\end{array}$ & $\begin{array}{c}690- \\
1,21 \mathrm{e} 3\end{array}$ & 0,5 & 100 \\
\hline
\end{tabular}

Evaluando la Tabla 3, claramente se evidencia que el mejor candidato para reemplazar al acero AISI 1030 son los dos aceros de baja aleación AISI 4135, los mismos que se muestran al final de la tabla respectivamente, esto debido a que cualquiera de estos dos ofrecen una relación aproximada de $K_{I c} / P=200$, lo cual resulta muy atractivo. Pero entre estos dos aceros, se elige finalmente al acero de baja aleación AISI 4135 templado y revenido, puesto que el valor del límite es mayor y ello optimiza aún más el material. A continuación, en la Tabla 4 se confronta las propiedades del acero AISI 1030 con el acero AISI 4135 seleccionado.

Tabla 4 Valores de rendimiento mejorado en el caso de un posible reemplazo del AISI 1030 por el AISI 4135, como material para las cuchillas del motocultor YTO DF151.

\begin{tabular}{|c|c|c|c|}
\hline & $\begin{array}{c}\text { Acero AISI } \\
1030\end{array}$ & $\begin{array}{l}\text { Acero AISI } 4135 \\
\text { templado y revenido }\end{array}$ & $\begin{array}{l}\text { Rendimiento } \\
\text { mejorado }\end{array}$ \\
\hline $\begin{array}{l}\text { Precio promedio } \\
\text { (Euro/kg) }\end{array}$ & 0,47 & 0,5 & $-6,40 \%$ \\
\hline $\begin{array}{c}\text { Tenacidad a la fractura } \\
\qquad\left(M P a^{*} m^{\wedge} 0,5\right)\end{array}$ & 42 & 100 & $138 \%$ \\
\hline Límite elástico $(\mathrm{MPa})$ & 620 & 950 & $53,23 \%$ \\
\hline Densidad $\left(\mathrm{kg} / \mathrm{m}^{\wedge} 3\right)$ & $7,85^{*} 10^{\wedge} 3$ & $7,83 * 10^{\wedge} 3$ & $0,25 \%$ \\
\hline
\end{tabular}

Todos los valores de rendimiento mejorado que se observa en la Tabla 4 , tienen signo positivo, excepto el valor con respecto al precio que tiene signo negativo, lo cual indica que el acero AISI 4135 resulta ser levemente más caro que el acero AISI 1030.

\section{Conclusiones •}

- Se concluye que el acero de baja aleación AISI 4135 templado y revenido, podría ser un firme candidato para poder reemplazar al acero AISI 1030, puesto que, aparte de cumplir con todas las restricciones (límite de fluencia mínimo especificado, dureza especificada, factibilidad para la forja, factibilidad para la soldadura térmica) 
requeridas para el diseño, también cuenta con el valor más alto de relación tenacidad a la fractura/precio $\left(K_{I c} / P=200\right)$, con lo cual se ha optimizado la selección del material, puesto que se a maximizado la tenacidad a la fractura y se ha minimizado el precio, dichos parámetro se consideran determinantes.

- Si bien es cierto es cierto, el acero AISI 4135 resulta ser un $6.4 \%$ (0.03 Euro $/ \mathrm{kg})$ más caro que el acero AISI 1030, este valor resulta despreciable si comparamos el rendimiento ganado en el resto de propiedades, que por obvias razones aumentaran la vida útil de las cuchillas.

- La diferencia de densidades (peso), no es un factor preponderante puesto que ambos aceros tienen prácticamente el mismo valor.

\section{Referencias bibliográficas.}

Gonzales, H., Pérez, W., Anaya, M., Restrepo, C., y Toro, A. (2007). Resistencia al desgaste de cuchillas de arados rotativos en operación en suelos tropicales, Scientcia et Technica Año XIII, 479-484.

Wilkinson, R., y Braunbeck, O. (1977). Elementos de maquinaria agrícola. FAO. Boletin de servicios agrícolas (12) FAO. Italia.

Hutchings, I. (1992). Tribolgy, Frictionand wear of engineering Materials (1th edition). Reino Unido: ed. Edward Arnold, a Vivison of hodder Headline PLC.

ASTM, (1987). Standard Terminology relating to wear and erosion, annual book of standars, Vol 03.02, p243-250.

Castillo, M.., y Bravo, P. (2017). Diseño y construcción de una máquina de desgaste acelerado para homologación de maquinaria agrícola en motocultorres para suelos típicos de la región centro del Ecuador: caso de estudio aspas del motocultor YTO 151 (Tesis de Maestría). Escuela Politécnica Nacional, Quito, Ecuador.

Ashby, M. (2011). Materials Selection in Mechanical Design (4th edition). Burlington, USA: Elsevier.

Muñoz, L. (2000). Estudio comparativo de la resistencia al desgaste abrasivo de una fundición nodular con microestructuras: bainíticas, perlíticas y martensíticas en la matriz. Universidad Nacional de Colombia.

Budynas, R., y Nisbett, J. (2011). Diseño en ingeniería mecánica de Shigley (4th edition). New York, USA:

McGraw-Hill.

Cañavate, O. (2014). Las máquinas agrícolas y su aplicación. Madrid, España: Uniprensa. 
Para citar el artículo indexado.

Abarca P, Castillo M., Bravo D., Sánchez F. \& Motúfar P. (2018). Selección de un material alternativo para el diseño y manufactura de cuchillas del motocultor YTO DF151, para trabajos en suelos de la región centro del Ecuador. Revista electrónica Ciencia Digital 2(3), 479-492.

Recuperado desde:

http://cienciadigital.org/revistacienciadigital2/index.php/CienciaDigital/article/view/170/15 $\underline{0}$

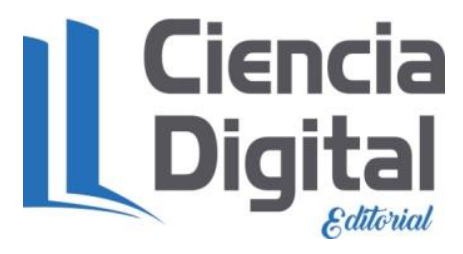

El artículo que se publica es de exclusiva responsabilidad de los autores y no necesariamente reflejan el pensamiento de la Revista Ciencia Digital.

El articulo queda en propiedad de la revista y, por tanto, su publicación parcial y/o total en otro medio tiene que ser autorizado por el director de la Revista Ciencia Digital.
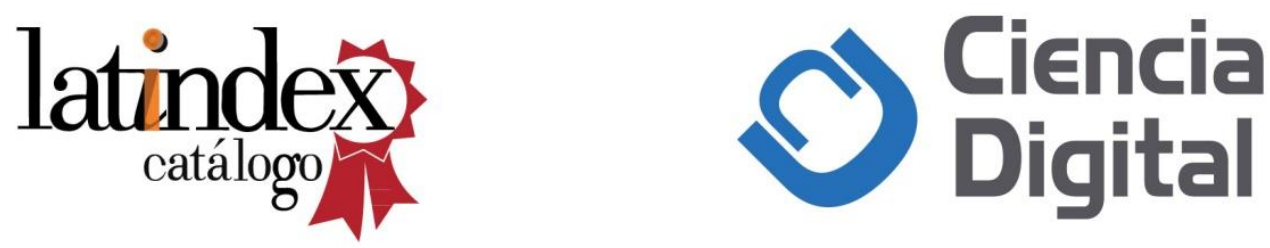